\title{
LES VINGT-CINQ ANS DU CENTRE
}

\author{
Rapport du Directeur au Conseil d'Administration \\ dans la séance du 28 mars $1950^{1}$.
}

\section{Monsieur le Président, Messieurs,}

La réunion d'aujourd'hui suit de près le vingt-cinquième anniversaire d'une première réunion où le Centre a été officiellement constitué. Vous ne vous étonnerez donc pas si, à cette occasion, je fais un retour en arriere. Mais auparavant, comme tous les ans, - car il n'est pas d'année où nous n'ayons eu des pertes à déplorer, - je commencerai par évoquer les membres de notre Conseil récemment disparus.

Il en est un qui, certainement, serait parmi nous, aujourd'hui, si la mort ne venait de le frapper très brusquement : le président Albert Lebrun, président d'honneur du Centre. Jusqu'à ces tout derniers temps, il avait conservé une étonnante vitalité; il voyageait, il se plaisait à faire des conférences : il aimait mieux, me disait-il l'an .dernicr, parler qu'écrire. Succédant à Paul Doumer et sachant tout ce que nous devions à son prédécesscur, il avait à cœur de nous témoigner de l'intérêt. Il nous suivait, de Ioin; mais, l'été dernier, il nous avait fait l'agréable surprise de venir à la séance de clôture de notre Semaine, d'y assister en auditcur attentif, et, à la fin, prenant la parole, de dire pour le Centre et pour son directeur des choses aimables que nous n'oublierons pas. Je n'ai point à vous retracer la carrière de ce brillant polytechnicien, de cet homme politique sage et conciliant, de ce bon Français. Vous me permettrez, à moi Lorrain, d'ajouter, pour ce Lorrain fidèle, des regrets personnels à l'hommage ému que lui doit le Centre.

Edouard Clavery, que nous avons perdu il y a près d'un an, était un vieil ami de notre maison. Figure originale, atta-

1. Publié sar la demande du Conseil. 
chante. Malgré la vieillesse et les infirmités, il ne manquait aucune de nos sćances de travail, et, quel que fût le sujet traité, il intervenait dans la discussion. Ancien ministre plénipotentiaire, il avait connu de lointains pays, il était curieux de la diversité humaine; mais il était curieux également des phénomènes de la vie universelle et, comme d'appartenir à notre Conscil, il s'honorait d'être membre perpétuel de la Socićté d'Astronomie. Il avait constitué au Centre un dossier - qui s'était ouvert avec le cours manuscrit de son maitre Brochard, pieusement conservé; qui s'était enrichi ensuite de brochures, sur des sujets variés, qu'il s'est plu à écrire jusqu’a ses derniers jours : on y peut recueillir des données démographiques, des rapprochements ingénieux de faits historiques, des pensées aussi, qui traduisent son être moral. "Là où un mortel vient en aide à un mortel, là est dieu », déclare-t-il; et encore, réflćchissant sur la fin de Thomme, \& quiconque a œuvré au profit du prochain peut et doit se dire, comme Horace : je ne mourrai pas tout entier».

Insérer son être spirituel dans la trame de la vie, nul ne l'a fait plus et mieux que cet autre membre que nous venons de perdre, Paul Boyer. Slavisant au large savoir, longtemps administratcur de l'Ecole des Iangues orientales vivantes, il n'a guère trouvé le temps d'écrire, il ne laisse pas d'œuvre égale à l'envergure de sa penséc; mais comme maitre, comme dirccteur, comme conseiller, il a exercé sur plusieurs générations une influence profonde; et les qualités de l'homme, accueillant, bienveillant, serviable, lui ont fait des amis de tous ceux qui l'ont approché.

uI Boyer était dejà des nôtres, ce jour de fin novembre 1924 où mon très cher ami Paul Doumer avait réuni à sa table douze personnalités, politiques et universitaires, pour faire du Centre de Synthèse, dont l'idée l'avait passionnément intéressé, une réalité officielle. De cette réunion, M. Edouard Herriot - alors président du Conseil - et moi, nous sommes les 'seuls survivants. C'aurait été une grande joic pour moi, s'il avait été libre, de voir aujourd'hui à mes côtés, comme président de notre Conseil, l'homme éminent dont l'amitié, comme celle de Paul Doumer est une des fiertés de ma vie. 
A la fin de ce déjeuner, Doumer me demanda de résumer pour les convives le programme élaboré au cours de deux années de méditations et d'entretiens, et dont je tenais dans mes mains la sixic̀me épreuve. Permettez-moi de rappeler, d'après des notes, la conclusion de mon exposé : « En prenant l'initiative que vous voulez bien encourager, je me laisse aller aux plus hautes ambitions - non pour moi, mais pour l'avenir de la science et de l'humanité. Pardonnez-moi ces grands mots; mais je suis, je veux être un idćaliste, un utopiste convaincu. Oui, j'ai les plus hautes ambitions; mais je prétends les réaliser peu à peu, sans tapage, avec prudence, avec méthode, avec suite. \$Et là-dessus Doumer, sollicitait l'appui et l'aide matérielle des personnalités présentes.

En 1929, après une installation provisoire rue Montpensier, au Palais-Royal, nous avons pris possession de l'Hôtel de Nevers, restauré grâce à de généreux concours. Et à la sćance dinauguration, comme plus tard à l'inauguration du buste qui maintient sa présence parmi nous, j'ai dit et redit les titres à notre reconnaissance de celui qui avait aplani pour nous toutes les difficultés et qui, partageant notre culte pour la Science, même lorsqu'il fut à l'Elysée, s'associait de loin - et quelquefois do près - à nos travaux.

La séance solennelle de 1929 m'a fourni l'occasion d'insister publiquement sur la raison d'être du Centre, sur le rôle de cette idée-force de synthèse dans la multiplicité des connaissances et le développement du spécialisme. J'ai souvent cité ce mot d'un savant : Partout on trouve les sciences, mais la Science nulle part $\gg$. Ici tout devait tendre à la Science, à la Science plénière. N'est-ce pas ici, dans le salon de Mme de Lambert, qu'est né l'esprit encyclopédique? Vous vous souvenez peut-être que, pour rendre hommage à ces précurseurs, nous avons, en 1932, sous le titre, L'Encyclopédic et les Encyclopédistes, organisé une exposition et des conférences. Mai 1932, c'est le moment où Doumer, qui se faisait une fête d'inaugurer notre exposition, nous a été enlevé par un crime sauvage et stupide. Cher Paul Doumer, homme de devoir et de bonté, Français incomparable, qu'on oublie trop, malgré les services qu'il a rendus - au-dedans et au dehors. Vous me pardonnerez, messieurs, si, dans ce jour d'anniversaire, je laisse monter le flot des souvenirs et aussi parler mon cour. 


\begin{abstract}
$* *$
Cette Synthèse, qui était notre objet, comment nous sommes-nous efforcés de la réaliser?

Le Centre, d'après ses statuts, comportait quatre sections.

Sur la base de la Revue de Synthèse historique, qui datait de 1900, et de l'Evolution de l'Humanité, qui datait de 1920, c'est la Scction de Synthèse historique, d'abord, qui s'est constituée solidement et s'est mise activement au travail. Nous avons fait le plan et commencé la réalisation d'un $R \dot{e}-$ pertoire historique pour la Synthèse; nous avons fait le plan, également, d'un Vocabulaire historique et, avant tout, discuté en séances les candidatures, en quelque sorte, des mots à y admettre. Ensuite, ce sont les mots eux-mêmes, traités par des collaborateurs qualifiés, qui ont été étudiés en des séances d'un intérêt très vif : l'étymologie de ces mots, l'évolution de leur sens ont donné lieu souvent à de curieuses remarques de psychologie collective. Après nous être attaqués, pour orienter l'ensemble du travail, à un certain nombre de termes importants, nous avons adopté l'ordre alphabétique pour hâter la publication d'un premier fascicule. Avant la guerre, nous tenions des séances tous les mercredis. Avec la guerre, l'occupation et ses suites, le travail est devenu plus intime : mes collaborateurs réguliers et moi, nous comptons bien que ce premier fascicule paraîtra avant la fin de la présente année.

La Revue de Synthèse historique intégrait dans l'Histoire - et, lors de son apparition, c'est ce qui a fait dire à certain juge excellent qu'elle * rendait un son nouveau » - toutes les histoires dites spéciales, c'est-à-dire les manifestations les plus diverses de l'activité humaine dans la vie de société; et elle intégrait aussi dans sa synthèse la protohistoire, la préhistoire, - domaines longtemps étrangers à ceux qui se considéraient comme les vrais historiens et pour qui archéologues, fouilleurs, explorateurs étaient des travailleurs d'un autre ordre. J'ai appelé Histoire militante celle qui se fait, non dans les bibliothèques et les archives, avec des livres et des parchemins, pour ainsi dire à huis clos, mais à travers le monde, à la conquête des restes de l'homme primitif et de ses œuvres, enfouis dans le sol. Dès notre installation à l'Hôtel de Nevers, le salon de Mme de Lambert, dans des conférences du soir, à entendu l'appel de savants éminents pour la belle aventure de la résurrection du passé humain.
\end{abstract}


Jacques de Morgan, - par d'admirables pages posthumes, Paul Pelliot, Eugène Pittard ont dit les peines et les joies de cette " histoire militante *.

Nous avons, d'autre part, dans des a Journées de Synthèse historique qui réunissaient des savants français et étrangers, associé étroitement la géographie et l'histoire, cherché à préciser le rôle des routes fluviales, de l'est à l'oucst, du nord au sud, dans le peuplement de l'Europe. Au cours de ces Journées, nous avons donné l'impulsion à des travaux cartographiques. Dans d'autres Journées, nous avons groupé archéologues, folkloristes et mythologues pour des études, manifestement synthétiques, qui ont été baptisées ici du nom d' * Archéocivilisation *.

Le Centre a essaimé : un certain nombre d'enquêtes, d'entreprises qui sont nées chez nous, se sont poursuivies au dehors. Nous ne pouvons que nous en féliciter; nous ne prétendons à aucun monopole.

Jusqu'ici, je ne vous ai parlé que de la Section de Synthèse historique - la fille ainée du Centre. De cette Section s'est disjointe l'Histoire des Sciences, à laquelle nous at tachons une particulière importance dans notre conception de la Synthèse historique. Nous avons marqué cette importance par la création d'une Section spéciale, -- avec une Bibliothèque, spéciale aussi, que d'heureuses circonstances nous ont procurée, avec son répertoire et, plus tard, sa Revue propres.

En même temps se développaient la Section des Sciences de la Nature et celle de Synthèse générale, et la Revue de Synthèse historique devenait Revue de Synthèse, organe du Centre, manifestation de son unité.

Et, en effet, si, pour suivre le progrès des connaissances dans les divers domaines, nous avions constitué des Sections distinctes, leur collaboration était constante. Le Vocabulaire historique, par exemple, qui comporte une grande variété de termes, faisait appel au concours de tous nos membres.

Tout tendait, en somme, et a de plus en plus tendu vers la Synthèse générale; et ce sont nos * Semaines de Synthèse », surtout, qui ont traduit cette convergence de nos efforts. Associant un public, toujours en grande partie compétent, à la discussion du sujet proposé, et publiẻes ensuite intégralement, sous leur forme vivante, elles ont étendu largegement notre action. La dernière Semaine, celle de 1949, était 
la quinzième; entre la onzième et la douzième, la guerre avait fait un vide de quatre ans.

Au début, nous avons traité les problèmes les plus variés, mais tous d'une grande généralité, impliquant tous l'intervention de savants venus de régions scientifiques diverses, et collaborant de façon directc, effective, à la solution du problème posé. «L'évolution en biologie », «La relativité 》, «L'évolution de la physique », "Science et Loi »; et, dautre part, "Civilisation », "Les origines de la Société », "L'individualité », «La Foule », "La notion de progrès devant la Science actuelle », * Le Ciel dans l'Histoire et dans la Science », "Le rôle de la Statistique », tels ont été nos premiers thèmes.

Mais, à un moment donné, nous avons voulu faire mieux encore : nous avons conçu une suite de Semaines liées, une Synthèse de Synthèses particulières. De 1937 à 1949, avec l'interruption tragique du libre travail en commun, c'est une explication complète du Récl, par la Science, que nous avons élaborée, - complète, sous la seule réserve du provisoire qu'implique le mouvement progressif, de plus en plus rapide, de cette Science.

En 1937, Semaine de l'Invention. En partant de cette donnée psychologique, la puissance créatrice de l'individu pensant, nous avons poussé la recherche de la tendance, d'un dynamisme également créateur, aussi loin que possible, au-delà même de la biologie, jusqu'aux problèmes que le biologiste pose au physicien.

En 1938, la Sensibilité dans l'Homme et dans la Nature. Notre objet consistait à mettre en évidence cette propriété qui fait que rien, dans la nature, n'est isolé, que tout retentit sur tout. Comme l'invention humaine, précédemment, la sensibilité humaine a été considérée sous tous ses aspects; mais, comme précédemment aussi, le réel a été étudié à tous ses degrés, dans ses analogies profondes.

Après avoir trouvé l'universel dynamisme lié à l'universelle sensibilité, nous devions nous demander ce que, dès lors, on doit entendre par a matière $>$. Tel a été le thème de 1939 : Qu'est-ce que la matière? L'histoire du concept a révélé que cet objet résistant n'avait été vraiment opposé comme substance étendue à une substance pensante qu'à partir du xvil ${ }^{\circ}$ siècle, avec Descartes; que la pensée primitive, et aussi la pensée réfléchie, tend au monisme : Descartes et ses disciples se sont débattus contre le dualisme initial. Et à travers 
les sciences actuelles, nous avons vu l'acquis positif rejoindre l'intuition première de l'humanité.

La matière n'étant qu'énergie au repos, il convenait de reprendre le problème par le dedans, et donc de suivre cette énergie, étofle mouvante du réel, dans ses transformations, jusqu'à son émergence vitale. C'est ce que nous avons fait, en 1946, avec l'Energie dans la Nature et dans la Vie.

En 1947, c'est l'émergence de cette énergie dans la conscience humaine que nous avons considérée. Après une revue de toutes les formes, de tous les degrés du psychisme, parvenus à la pensće, nous y avons trouvé l'être : Cogito ergo sum. A la mode de « l'existentialisme » nous opposions le témoignage essentialiste de la conscience, la révélation de l'Etre universel qui y est contenue. Valeur philosophique de la Psychologie, tel était le titre de notre Semainc.

Toutes les sciences - représentées par les plus compétents et souvent les plus éminents d'entre les savants - ont concouru à former cette notion de l'Univers, un dans son cssence, infiniment divers dans ses aspects, ascensionncl dans ses transformations, et dont la cîme est dans la Pensée. L'Univers s'apparaît et se pense par la Science. La Synthèse des connaissances, c'est la Philosophie. Je dirais volonticrs : c'est la révélation.

En 1948, nous avons fait une sorte de vérification de cette philosophie, en passant de l'abstrait au concret. Naissance de la Terre et de la Vie sur la Terre : tel était notre thème. Evocation cosmogonique: dans l'infini des galaxies, le système solaire; dans le système solaire, l'évolution de notre planète; puis les tâtonnements, et les réussites de la vie : voilà qui nous a montré en action cet énergéisme d'où provient l'humanité. Et, dans une dernière séance, nous avons abordé les problèmes de l'avenir de la Terre et de la pluralité des Mondes.

L'année dernière, enfin, pour ètablir une relation entre notre quinzième Semaine et notre quart de siècle de vie et de travail, nous avons traité ce sujet : La Synthèse, idée-force dans l'évolution de la pensée. Au terme de cette Semaine vous pardonnerez à mon âge, à ma continuité d'effort - une brève apparition du moi - j'ai montré comment avait germé en mon esprit l'idée du Centre. Depuis mon premier livre, Vie et Science, à travers ma thèse, La Synthèse des connaissances et l'Histoire, puis la Reoue et la Bibliotheque de Synthèse historique, j'avais été animé, et par la foi en la Science, et par 
la conviction que la Science, pour une explication totale, demande à être située, intégrée dans l'Histoire ${ }^{2}$.

II y a des étapes, dans cette recherche de la Vérité, qui ont un intérêt majeur. A Descartes, à notre maître Descartes, nous avons, en 1948 et cette année encore, consacré des Journies, où ont été mis en lumière les multiples aspects de sa pensée, son inépuisable influence, sa modernité étonnante. Je vous ai dit ce que nous avons fait pour les Encyclopédistes, pour Diderot et Condorcet, ces grands précurseurs.

«En somme, ce que l'histoire humaine, en continuité avec celle de la Vie, fait apparaitre, fait éclater, n'est-ce pas qu'au cours des âges s'est préparé, façonné, perfectionné pcu à pcu un merveilleux instrument, fragile, et cependant d'un pouvoir presque illimité : l'esprit. L'Histoire, en vérité, est comme une introspection collective, où l'Humanité prend conscience de sa poussée interne, des conditions de son développement passé, des directions de son développement futur ${ }^{3}$.

Cantonnés, pour notre part, dans cette recherche de la vérité spéculative, nous nous sommes toujours refusés à intervenir, de façon directc, dans la vie du présent. Toutefois, le Centre a prêté son concours à une entreprise destinće, non à vulgariser, mais à diffuser la science et à en répandre le culte : je veux parler du journal Science, avec son feuilleton d'Encyclopédie périodique. Interrompu par la guerre, ce journal, qui avait été accueilli avec une ferveur touchante par un public d'instituteurs, de chercheurs de foi, a dû être abandonné.

Toute cette cuvre, à laquelle tant de personnes se sont vouées ou intéressées, vous est apparue - à part moi - anonyme. Si je vous énumérais tous ceux qui, d'une façon ou d'une autre, lui ont apporté leur concours, tous ccux. notamment, qui ont participé à nos Semaines, la liste glorieuse en serait interminable. Je me contenterai d'évoquer des morts : parmi les fondateurs, avec Paul Doumer en première ligne, Raymond Poincaré, Paul Appell, Maurice Croiset, Cavalier, Charléty, Roland Marcel; parmi les collaborateurs intimes, Abel Rey et Paul Langevin.

Messieurs, les résultats spirituels que nous avons obtenus sont d'autant plus méritoires, sans doute, que nos ressources

2. On trouvera plus loin cette $15^{\circ}$ * Semaine * intégralement reproduite.

3. Paroles de 1931. 
matérielles, qui ont toujours été médiocres, ont faibli encore ces dernières années. Il n'est pas de réunion de notre Conseil où je ne vous aie fait part de nos difficultés, où je n'aie déploré les retards de nos publications. C'est gràce à tant de concours bénévoles, et au dévouement de nos collaborateurs réguliers, que nous avons accompli l'œuvre que je vous ai retracée. Nous sommes privés actuellement de ces jeunes attachés, étudiants besogneux pour qui, jadis, une faible rémunération n'était pas méprisable et qui trouvent, aujourd'hui, des aides plus efficaces.

La création de l'Unesco, à Paris, posait pour nous un problème. Ne pourrions-nous, en nous affiliant, d'une façon quelconque, à l'institution nouvelle, en tirer quelque avantage? Voici le résultat de nos réflexions. Il est certain que l'Unesco peut rendre de grands services pour répandre la culture et unifier l'éducation. Mais, en ce qui concerne la Science, des entreprises nombreuses, diverses, mais sans coordination intime, des idées comme celle d'une association internationale des Universités, des. congrès de toutes sortes, c'est autre chose que ce que nous avons voulu et réalisé : le travail en commun, permanent, pour unifier les connaissances et éclairer l'avenir humain. Réflexion faite, nous avons voulu conserver notre autonomie.

En ce qui concerne les Congrès, j'ai eu souvent l'occasion de dire et d'écrire ce que j'en pense. Il va de soi que le contact qu'ils établissent, les échanges d'idées qu'ils provoquent, les sympathies qu'ils font naître entre des personnalités, souvent de pays divers, toujours de formations, de mentalités diverses, ont une réelle utilité. Mais de l'objet de ces réunions, que faut-il penser? S'il s'agit d'une science particulière, si l'on veut simplement contrôler des résultats positifs, préciser des questions de méthode, en un mot promouvoir cette science spéciale, on ne saurait donter qu'il y ait profit au rapprochement, à la confrontation des compétences. Ou encore, s'il s'agit de problèmes moraux, - liberté, pacifisme, humanismc, quoique le profit ne soit pas, généralement, en proportion du temps et des paroles qu'on y consomme, quelques moments d'entente, et parfois d'émotion commune, peuvent avoir un effet bienfaisant. Mais quand il s'agit de philosophie, soit que, selon la formule traditionnelle, la métaphysique se mêle au savoir positif, soit que, sous le titre de philosophie des sciences, on juxtapose les sciences de la nature, à l'exclusion des sciences humaines, - et même en supposant toutes les scien- 
ces représentées, -- comme on est loin de cette préoccupation de Synthèse, qui inspire nos Semaines!

Je ne fais pas ces critiques ou ces réserves par un vain désir de souligner l'originalité de notre rôle. Mais, à cette réunion d'anniversaire qui, selon toutes probabilités, ne sera pas suivie, pour moi, de beaucoup d'autres, parlant devant ceux qui continueront à représenter le Centre, j'ai voulu en faire ressortir l'esprit. Vous me permettrez d'exprimer le vœu que cet esprit me survive.

"Je ne connais qu'un seul résultat à la science, a dit Renan, c'est de résoudre l'énigme; c'est de dire définitivement à l'homme le mot des choses. » Ce propos de jeunesse, que Renan vieilli n'aurait peut-être pas confirmé, nous l'avons fait nôtre. Et je veux terminer en rappelant ce que je disais, en 1931, dans une circonstance pour moi mémorable : « Ici, notre oeuvre consiste à faire resplendir la beauté, à faire sentir l'attrait de la science pure, - mais de la science pleine, et qui doit se tourner en maîtresse de vie... Il n'y a pas de plaisir plus vif, — au vrai sens de ce mot, - pas de sentiment plus complet $d u$ vouloir-vivre universel que ce qu'éprouve le savant quand il cherche et résoud des problèmes, le philosophe quand il pense et systématise. La parole sublime que Pascal prête à Jésus : "Tu ne me chercherais pas sí tu ne "m'avais déjà trouvé " s'applique ici. L'ardeur qui anime l'homme de science est la même qui brûle le cœur du croyant. La foi qui a élevé jadis les émouvantes cathédrales est la même qui a bâti l'édifice spirituel de notre Centre et qui dresse, dans ce lieu «inspiré », sa flèche de synthèse. 》 